\title{
Quasi-periodic Oscillations and Noise in Accreting Black Holes and Low-magnetic Field Neutron Stars
}

\author{
M. van der Klis
}

Astronomical Institute "Anton Pannekoek", University of Amsterdam, and Center for High-Energy Astrophysics, Kruislaan 403, 1098 SJ Amsterdam, The Netherlands

\section{Introduction}

The X-ray spectrum and the rapid X-ray variability of accreting compact objects have a common origin, and their properties can therefore be expected to be coupled. Indeed, it turns out that the X-ray spectrum and the power spectrum show correlated variations, which occur as a function of variations in the mass flux $\dot{M}$.

Stellar mass black holes and neutron stars have similar mass and size, and therefore their accretion phenomena may be expected to show similarities. Indeed, similarities exist that indicate that a unified description may be possible. If a particular phenomenon is seen in both neutron star and black-hole candidate systems this shows immediately that it cannot be due to any property that is unique to either neutron stars or black holes, such as the presence or absence of a surface, or of a strong non-aligned magnetic field. The quest for characteristics that are unique to black holes continues - I shall mention a few candidates below.

The power spectra of accreting compact objects can be described in terms of a small number of simple shapes (see van der Klis 1995a, c). Power law noise has a power distribution $\propto \nu^{-\alpha}$, band limited noise one that steepens towards high $\nu$ and flattens towards low $\nu$. Band limited noise that has a maximum at $\nu>0$ is called peaked; if the maximum is at $\nu=0$ the component is called flat-topped. The same power spectral component can be at one time flat-topped and at another time peaked. Quasi-periodic oscillations (QPO) are a type of peaked noise. Usually, the term QPO is reserved for relatively narrow peaks $(\Delta \nu / \nu \lesssim 0.5)$.

\section{$2 \mathrm{Z}$ and atoll sources}

$\mathrm{Z}$ and atoll sources (Hasinger and van der Klis 1989, hererafter HK89) are low magnetic-field neutron stars. Only a summary of their properties is presented here (see further van der Klis 1989, 1995c). The X-ray spectral changes are usually subtle, and colour-colour diagrams (CDs) and hardness-intensity diagrams 
(HIDs), plots of X-ray hardness ratios vs. each other or vs. count rate are used to describe the $\mathrm{X}$-ray spectral variations. The sources produce characteristic tracks in the CD/HIDs, and source position in the track is governed by $\dot{M}$.

The six known $Z$ sources produce Z-shaped tracks. Z source power spectra show three broad noise components, very low frequency noise (VLFN), low frequency noise (LFN) and high frequency noise (HFN) and two QPO components, horizontal branch oscillations (HBO) and normal and flaring branch oscillations (N/FBO). HBO and LFN are a QPO and a band limited noise component that appear an disappear together, and are likely physically related. They are strongest at low $\dot{M}$ and disappear at high $\dot{M}$. HBO frequency $(13-55 \mathrm{~Hz})$ and LFN cut-off frequency $(2-20 \mathrm{~Hz})$ increase with $\dot{M}$. LFN can be flat topped or peaked, depending on the source. HBO and LFN are well explained in the magnetospheric beat frequency model (Alpar and Shaham 1985, Lamb et al. 1985), which requires $\mathrm{Z}$ sources to have a magnetosphere. Some pulsars show QPO that may be caused by a similar mechanism (Angelini et al. 1989, Finger 1995). N/FBO have a preferred frequency near $6 \mathrm{~Hz}$. In Sco X-1 and GX 17+2, their frequency has been observed to increase from $\sim 6$ to $\sim 20 \mathrm{~Hz}$ when $\dot{M}$ increases. Radiation forces at near-Eddington accretion rates probably play the key role in their formation (van der Klis et al. 1987, Hasinger 1987, Lamb 1989, Fortner et al. 1989, Miller and Lamb 1992, Alpar et al. 1992).

The 12-15 known atoll sources (HK89, van der Klis 1995c) show a curved track, that is often fragmented due to observational effects. increases. Their power spectra show two broad noise components called very-low-frequency noise (VLFN) and high-frequency noise (HFN). Atoll source HFN has a cut-off frequency of $0.3-20 \mathrm{~Hz}$ and depends strongly on $\dot{M}: \mathrm{rms}$ amplitude falls from $>20 \%$ to $<2 \%$ and the cut-off frequency increases when $\dot{M}$ increases (Yoshida et al. 1993, Prins et al. 1995). Atoll HFN is sometimes flat-topped and sometimes peaked.

HK89 proposed that the neutron stars in atoll sources have lower magnetic field strengths than $\mathrm{Z}$ sources, and are constrained to lower $\dot{M}$. The lower field explains why the (magnetospheric) HBO are not seen in atoll sources, and the lower $\dot{M}$ why the same is true for the (near-Eddington) N/FBO. The implied relation between $\dot{M}$ and magnetic field strength may have an evolutionary origin (van der Klis 1991). Predictions are that an atoll source that becomes bright will show $\mathrm{Z}$ source high $\dot{M}$ properties (N/FBO and appropriate spectral branches), but never $\mathrm{HBO}$, and that a $\mathrm{Z}$ source that becomes faint will show millisecond pulsations. The properties of Cir X-1 fit the first prediction. The source is apparently an example of an atoll source that can reach $\dot{M}_{E d d}$ (van der Klis 1991; Oosterbroek et al. 1995). As will be discussed in Section 4 Cir X-1 also shares some characteristics with black-hole candidates.

\section{Black hole candidates}

Three source states are distinguished in black-hole candidates (Fig. 1). In the low state (LS) the X-ray spectrum is a flat power law with photon spectral 
index $1.5-2$. In the high state (HS) the $1-10 \mathrm{keV}$ flux is much higher due to a soft component; the power law sometimes "sticks out" from under the soft component $>10 \mathrm{kev}$. In the (rare) very high state (VHS) a similar (but brighter) soft component is seen, with perhaps an additional power law component. The VHS mainly differs from the HS by the properties of its rapid X-ray variability.

BLACK-HOLE-CANDIDATE POWER SPECTRA

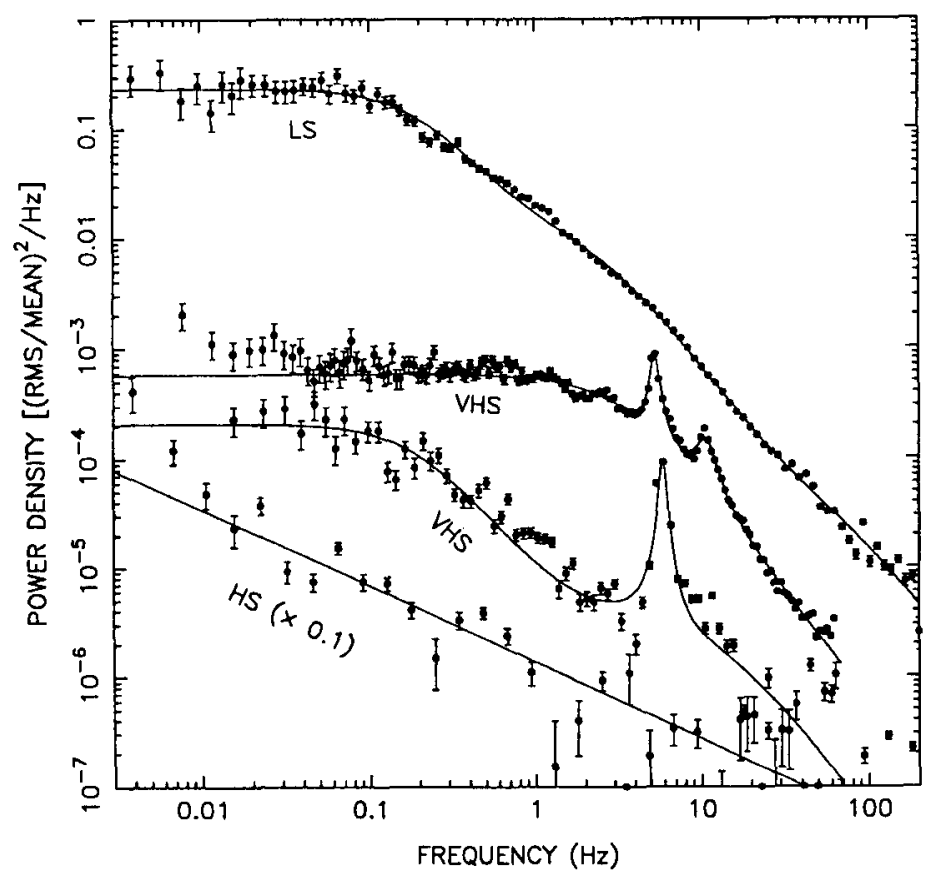

Fig. 1. Power spectra from Ginga data of black-hole candidates in the low (LS; Cyg X-1), high and very high (HS and VHS; GS 1124-68) states.

The LS shows strong (30-50\% amplitude) band-limited noise with $\nu_{\text {cut }}$ between 0.03 and $0.3 \mathrm{~Hz}$ which is usually flat-topped, but sometimes peaked (Vikhlinin et al. 1994). The level of the flat top and the cut-off frequency $\nu_{\text {cut }}$ vary in anticorrelation, sometimes while the power spectrum above $\nu_{\text {cut }}$ remains unchanged (Belloni and Hasinger 1990, Miyamoto et al. 1992a). In the HS variability is weak. Slow QPO with frequencies similar to the LS noise cut-off frequencies ( $\sim 0.08-0.8 \mathrm{~Hz}$; Motch et al. 1983, Ebisawa et al. 1989, Grebenev et al. 1991) and possibly related to peaked LS noise sometimes occur in LS and HS. The rare VHS shows $3-10 \mathrm{~Hz}$ QPO and rapidly variable broad-band noise. CD/HID branches occur in the VHS, and the power spectral parameters seem to depend 
on position in the branches, but these branch structures are not very similar from one epoch to the next ("messy" branches). The LS and VHS band limited noise cut-off frequency and amplitude fit one relation (van der Klis 1994b), suggesting that they form one phenomenon. The transient black-hole candidate GS 1124-68 (Nova Mus 1991 = GU Mus) in its decay went through all three states (Miyamoto et al. 1992b), strongly suggesting that the states directly follow $\dot{M}$. However, recent evidence indicates that some black hole transients, even when they are very luminous, remain in the "low" state; see Section 5.

\section{Similarities between black hole candidates and low magnetic field neutron stars}

There is a number of striking similarities between black-hole candidate and neutron star phenomenology (see van der Klis 1994a, 1995a). The black-hole candidate LS is very similar to the atoll source low $\dot{M}$ ("island") state. Both states occur at the lowest $1-10 \mathrm{keV}$ count rates and inferred $\dot{M}$ levels. Both are dominated by strong (several 10\%) band limited noise (LS noise and atoll HFN) which is sometimes flat-topped and sometimes slightly peaked. Sometimes the power spectra are nearly indistinguishable (Fig. 2, top; see also Yoshida et al. 1993), and the atoll source $13-80 \mathrm{keV}$ X-ray spectra at low $\dot{M}$ become as hard as in blackhole candidates in the LS (Van Paradijs and van der Klis 1994). Z source LFN is also similar to black-hole candidate LS noise and atoll HFN: all 3 components are stronger at lower $\dot{M}$, disappear at higher $\dot{M}$, can be peaked and flat-topped, and have a higher $\nu_{\text {cut }}$ at higher $\dot{M}$. The absence of a similar band-limited noise component in pulsars suggests that such noise arises through inhomogeneities in the inner disk, absent in pulsars. This is in accordance with the explanation of the LFN in the beat-frequency model. The black hole candidate VHS has strong similarities to the $\mathrm{Z}$ source high $\dot{M}$ ("normal/flaring branch") state. Both occur at the highest inferred $\dot{M}$ levels, and both show QPO, with similar frequencies $(6-20 \mathrm{~Hz}$ in the neutron star systems, $3-10 \mathrm{~Hz}$ in the black-hole candidates), that depend on the position of the source in branched tracks in the HID/CDs. Clearly different is the harmonic content of the QPO (black-hole candidate VHS QPO show strong harmonics, Z source N/FBO do not) and the character of the HID/CD branches (much "messier" in BHCs). Another difference is that Z sources do not show the rapidly variable broad band noise seen in black-hole candidates.

The properties of Cir X-1 provide a further link between neutron stars and black holes. In some of its high states (Tennant 1987, Makino et al. 1992, Oosterbroek et al. 1995), this source shows a mix of $\mathrm{Z}$ source and black-hole candidate high $\dot{M}$ characteristics (see Fig. 2). It shows QPO with frequencies between 6 and $20 \mathrm{~Hz}$ and no second harmonics (both $\mathrm{Z}$ source characteristics) in combination with messy branches in the CD/HID and rapidly variable broad band noise (BHC characteristics). The reason, then, that Cir X-1 sometimes resembles a black hole in its rapid variability characteristics, (e.g. Toor 1977, Samimi 1979), while its X-ray bursts (Tennant et al. 1986a, b) show it to be a neutron 


\section{BLACK HOLE}
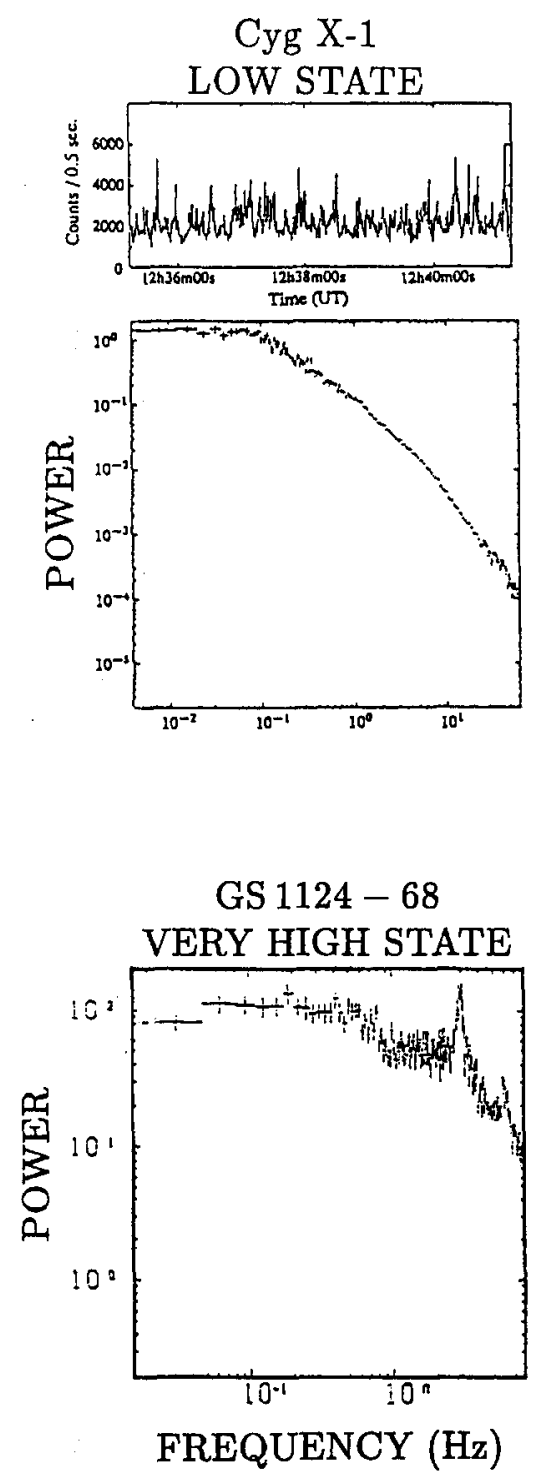

\section{NEUTRON STAR}

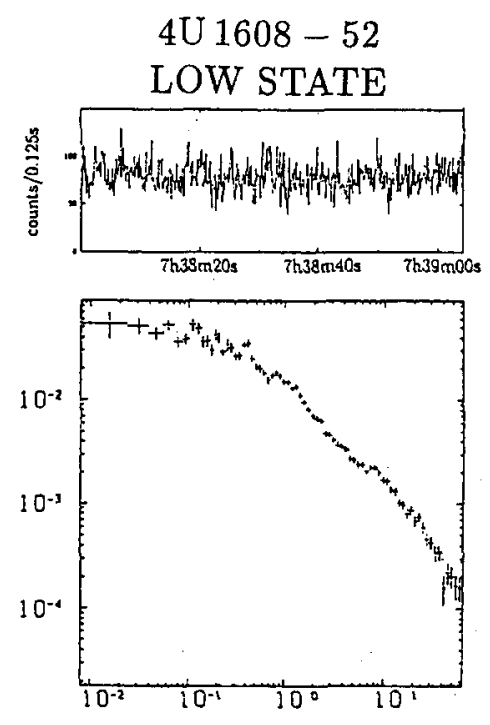

Cir X-1

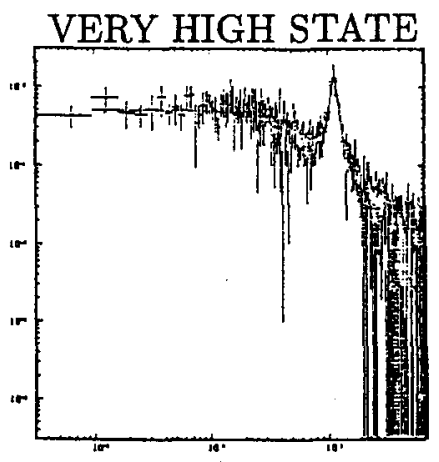

FREQUENCY $(\mathrm{Hz})$

Fig. 2. Power spectra from the black-hole candidates Cyg X-1 (top left) and GS 1124-68 (bottom left) in the low state and the very high state, respectively, and from the low magnetic-field neutron stars 4 U 1608-52 (top right) and Cir X-1 (bottom right) in the atoll island state and a very high $\mathrm{X}$-ray brightness state, respectively, illustrate the similarity between neutron star and black-hole candidate low and very high states. Compiled from Inoue (1992), Takizawa et al. (1995) and Makino et al. (1991) 
star, is that it is the only neutron star that we know that has a magnetic field as low as in atoll sources that sometimes accretes at near-or super-Eddington rates. Cir X-1 is therefore a key object, as it can help to distinguish between phenomena that are characteristic for accretion onto any compact object that has no appreciable magnetic field, and phenomena that are truly characteristic for accretion onto a black hole. From its properties one may conclude that a high harmonic content of the high $\dot{M}$ QPO may be a black hole signature, whereas rapidly variable broad band noise and messy branches are not.

On the basis of this array of similarities, it can be concluded that the phenomenology of the black-hole candidates and low magnetic-field neutron stars may be described in terms of three $\dot{M}$-driven states that are common to accreting low magnetic-field neutron stars and accreting black holes (van der Klis 1994a). Fig. 3 presents a line-up of the three common states of black-hole candidates and low magnetic-field neutron stars.

\section{Inclination effects}

Detailed examination of the properties of Z sources, in particular in their flaring branches, has led Kuulkers and van der Klis (1995) to propose that obscuration by a geometrically thick inner accretion disk plays a role in $\mathrm{Z}$ source phenomenology. The disk swells when $\dot{M}$ increases, and for higher inclination $i$ obscuration effects already set in at lower $\dot{M}$. A similar model might apply to black-hole candidates (van der Klis 1994a, 1995b). The reason for the disappearance of the hard LS X-ray spectral component in the HS may be obscuration of a central, hot and rapidly variable region by a puffed-up accretion disk. Some black hole transients, such as GS $2023+338$, show only a hard power-law X-ray spectral component, even when they are very bright. This might in this model be due to a pole-on viewing geometry. In GX 339-4 the observable energy flux in the 1-200 keV band is higher in the low state than in the high state (Grebenev et al. 1993). The increasing concentration of the hard X-rays towards the (rotation) polar axes with increasing $\dot{M}$ might explain this: at high $\dot{M}$ most of the energy would be leaving the system along the polar axis and would, in a high-inclination source, not be seen by us.

In the low magnetic-field neutron stars the X-ray flux is an unreliable indicator of $\dot{M}$; the same might turn out to be the case in the black-hole candidates. Note, that the mass flux $\dot{M}$ that by hypothesis determines source state is the mass flux towards the compact object. At near- and super-Eddington rates, not all of this matter may actually accrete; jets might for example be formed when $\dot{M}$ becomes high enough.

\section{Conclusion}

The millisecond fluctuations in black holes, and in neutron stars with various magnetic field strengths can perhaps be understood in common terms, as follows. 


\begin{tabular}{|c|c|c|c|c|}
\hline $\begin{array}{r}\text { Phenomena } \\
\text { Guess at B } \\
\end{array}$ & $\begin{array}{c}\text { Black hole } \\
\text { (0 Gauss) }\end{array}$ & $\begin{array}{c}\text { Atoll source } \\
\left(\leqslant 10^{9-10} \text { Gauss }\right) \\
\end{array}$ & $\begin{array}{c}\text { Z source } \\
\left(\sim 10^{9-10} \text { Gauss }\right) \\
\end{array}$ & Guess at $\dot{M}$ \\
\hline High $\dot{M}$ QPO & $\left.\right|_{\text {VHS }}$ & $\begin{array}{c}\text { Cir X-1 } \\
\text { (high state) }\end{array}$ & $\stackrel{\text { FB }}{t}$ & $z^{1} \dot{M}_{E}$ \\
\hline $\begin{array}{l}\text { Weak power-law } \\
\text { noise }\end{array}$ & & $\uparrow_{\text {Banana }}^{\uparrow}$ & NB & $\sim 0.9 \dot{M}_{E}$ \\
\hline $\begin{array}{l}\text { Weak power-law } \\
\text { noise }+ \\
\text { band-limited } \\
\text { noise }\end{array}$ & $\stackrel{\text { HS }}{1}$ & & $\underset{\mathrm{HB}}{\uparrow}$ & $\sim 0.5 \dot{M}_{E}$ \\
\hline $\begin{array}{l}\text { Strong } \\
\text { band-limited } \\
\text { noise }\end{array}$ & LS & $\begin{array}{l}\text { Island } \\
\text { (most bursters } \\
\text { and dippers) }\end{array}$ & $\begin{array}{l}\text { (ms X-ray } \\
\text { pulsars?) }\end{array}$ & $\sim 0.01 \dot{M}_{E}$ \\
\hline
\end{tabular}

VBS: very high state; HS: high state; LS: low state; FB: flaring branch; NB: normal branch; HB: horizontal branch.

Fig. 3. Proposed classification scheme for X-ray binary source states. There are three states that are common to neutron stars and black holes; in a given source the mass transfer rate $\dot{M}$ towards the compact object determines the state. The power spectral shapes that are characteristic of each state are indicated at left. The conventional names of the source states in each source type are indicated. Magnetic field strengths and mass fluxes are rough indications only; other source parameters, such as inclination, might affect the $\dot{M} / \dot{M}_{E}$ levels at which state transitions occur.

Two structures determine the basic physics of the accretion process, namely the magnetosphere and the inner (radiation pressure dominated) disk. Z sources are the most complex, showing $\mathrm{HBO}$ and $\mathrm{N} / \mathrm{FBO}$, as well as LFN and HFN, because their field is weak enough to allow the presence of an inner disk (like in black holes and atoll sources) and strong enough to form a magnetosphere (like in pulsars, but much smaller). Black holes and atoll sources have an inner disk and no appreciable magnetosphere and therefore only show an N/FBO-like component (VHS QPO) and an LFN-like component (LS noise). X-ray pulsars (not 
discussed here) have a magnetosphere and no inner disk and therefore show only an $\mathrm{HBO}$-like and an HFN-like component. Inner disk structure causes anisotropic emission, and thereby inclination effects are introduced in the phenomenology.

Acknowledgements: This work was supported in part by the Netherlands Organization for Scientific Research (NWO) under grant PGS 78-277.

\section{References}

Alpar M.A., Shaham J., 1985, Nature 316, 239

Alpar M.A. et al., 1992, A\&A 257, 627

Angelini L., Stella L., Parmar A.N., 1989, ApJ 346, 906

Belloni T., Hasinger G., 1990, A\&A 227, L33

Ebisawa K., Mitsuda K., Inoue H., 1989, PASJ 41, 519

Finger M., 1995, Proc. IAU Symp. 165, The Hague, August 1994, in press

Fortner B., Lamb F.K., Miller G.S., 1989, Nature 342, 775

Grebenev S.A. et al., 1991, Sov. Astron. Lett. 17(6), 413

Grebenev S. et al., 1993, A\&A Suppl. 97, 281

Hasinger G., 1987, A\&A 186, 153

Hasinger G., van der Klis, M., 1989, A\&A 225, 79 [HK89]

Inoue H., 1992, in Accretion Disks in Compact Stellar Systems, J.C. Wheeler (ed.), ISAS RN 518

Kuulkers E., van der Klis, M., 1995, A\&A, in press

Lamb F.K., 1989, Proc. 23rd ESLAB Symposium, ESA SP-296, 215

Lamb F.K. et al., 1985, Nature 317, 681

Makino Y., Kitamoto S., Miyamoto S., 1991, poster presented at the 28th Yamada Conference, Nagoya, Japan, April 8-12 1991

Makino Y., Kitamoto S., Miyamoto S., 1992, in Frontiers of X-ray astronomy, Tanaka and Koyama (eds.), Universal Academy Press, Tokyo, p. 167

Miller G.S., Lamb F.K., 1992, ApJ 388, 541

Miyamoto S. et al., 1992a, ApJ 391, L21

Miyamoto S. et al., 1992b, Proc. Ginga Memorial Symp., Makino and Nagase (eds.), ISAS, Tokyo, p. 37

Motch C. et al., 1983, A\&A 119, 171

Oosterbroek T. et al., 1995, A\&A, in press

Prins S. et al., 1995, A\&A, in preparation

Samimi J. et al., 1979, Nature 278, 434

Takizawa M. et al., 1995, in press

Tennant A.F., 1987, MNRAS 226, 971

Tennant A.F., Fabian A.C., Shafer R.A., 1986a, MNRAS 219, 871

Tennant A.F., Fabian A.C., Shafer R.A., 1986b, MNRAS 221, 27p

Toor A., 1977, ApJ 215, L57

Van der Klis M., 1989, ARA\&A 27, 517

Van der Klis M., 1991, NATO Advanced Study Institute 344, 319

Van der Klis M., 1994a, ApJ Suppl. 92, 511

Van der Klis M., 1994b, A\&A 281, L17

Van der Klis M., 1995a, NATO Advanced Study Institute 450, 301

Van der Klis M., 1995b, Proc. COSPAR Meeting on Galactic and Extragalactic Black Holes, Hamburg, July 1994, in press 
Van der Klis M., 1995c, AIAP preprint 1993-009; in X-Ray Binaries, Lewin, van Paradijs and van den Heuvel (eds.), Cambridge University Press, in press

Van der Klis M. et al., 1987, ApJ 316, 411

Van Paradijs J., van der Klis M., 1994, A\&A 281, L17

Vikhlinin A. et al., 1994, A\&A 287, 73

Yoshida K. et al., 1993, PASJ 45, 605

D. Dravins: As you mentioned, the relevant dynamic timescale for black-hole systems may be in the range $0.1-1$ milliseconds, potentially observable as variability at kilohertz frequencies. Is it possible to make any extrapolation or estimates of what phenomena one could observe there, once the observational capacity becomes available?

M. van der Klis: It is conceivable that in black holes we might be able to detect the signature of matter in its last stable orbit at $3 R_{S}$. However, it is very hard to predict how strong the signal would be. In $1995 \mathrm{NASA}$ will launch XTE, which for bright $X$-ray sources will detect count rates up to about $10^{5}$ cts; this we hope will make it possible to detect such a signal, if it is there. 